\title{
Mixed genetic and environmental sex determination in an androdioecious population of Mercurialis annua
}

\author{
JOHN PANNELL* \\ Department of Plant Sciences, University of Oxford, South Parks Road, Oxford OX1 3RB, U.K.
}

\begin{abstract}
Mercurialis annua is a ruderal of pan-European distribution. In southern Spain, southern Portugal and northern Morocco, males coexist with monoecious (cosex) individuals at frequencies which vary between zero and about 30 per cent. Here, I report the results of two glasshouse experiments which aimed to characterize the mode of sex determination in one such androdioecious population. In a breeding experiment, cosexes isolated from males produced only cosexual progeny, whereas those allowed to mate with males produced both cosexual and male offspring. The results are consistent with a single-locus model of sex inheritance, with maleness determined by a dominant allele. In a density experiment, the frequency of males correlated positively with density, confirming field observations. Differential mortality or germination between the sexes was excluded by experimental design, so that the result supports the hypothesis of density-dependent gender choice. A negative correlation between density and the proportion of 'late cosexes' (males which turned cosexual late in their development), and the lack of any differences in the proportion of pure (unchanged) cosexes between density, suggest that only males were capable of sex change. This interpretation is consistent with the results of the breeding experiment, in which late cosexes occurred only in the offspring of cosexes allowed to mate with males. I argue that these findings help to explain the maintenance of androdioecy in $M$. annua.
\end{abstract}

keywords: androdioecy, dioecy, reproductive assurance, sex change, sex choice, sexual dimorphism.

\section{Introduction}

In sexually dimorphic (e.g. dioecious, androdioecious or gynodioecious) plant species, gender may be determined genetically, environmentally, or by a genotype-environment interaction (Charnov, 1982; Lloyd \& Bawa, 1984; Meagher, 1988). Some genetic systems of sex determination in dioecious species appear to be chromosomal in nature (reviewed in Westergaard, 1958; Meagher, 1988). Others involve the expression of genes at one or more loci on chromosomes which cannot be identified cytologically. In diploid, dioecious populations of Mercurialis annua, for example, sex is determined by the interactive effects of dominant or recessive sex alleles at three independently segregating loci (Irish \& Nelson, 1989; Durand \& Durand, 1991b; see below).

*Present address: Department of Botany, University of Toronto, 25 Willcocks Street, Toronto, Ont., Canada M55 3B2. E-mail: Pannell@botany.utoronto,ca
In gynodioecious species (i.e. where females coexist with cosexes), male sterility is frequently the outcome of interactions between nuclear and maternally inherited cytoplasmic genes (e.g. Gouyon \& Couvet, 1987; Durand \& Durand, 1991a; Van Damme, 1991), whereas female sterility in androdioecious species (i.e. where males coexist with cosexes) is most likely to be controlled solely by nuclear genes (paternal inheritance of cytoplasmic genes is uncommon in Angiosperms; Reboud \& Zeyl, 1994). In the androdioecious notostracan clam shrimp Eulimnadia texana, sex is inherited at a single locus, with a recessive allele determining maleness (Sassaman \& Weeks, 1993). However, this study of Mercurialis annua presents the first attempt to elucidate the genetic system of sex determination for an androdioecious plant species.

Environmental factors are also known to influence sex ratios in several plant species (Lloyd \& Bawa, 1984; Meagher, 1988), with males most typically tending to be over-represented in resource-limited 
or less favourable environments (reviewed in Freeman \& Vitale, 1985; Meagher, 1988). This has been attributed both to lower mortality rates of males than females under less favourable conditions, as well as to environmental influences on sex expression itself (Sakai \& Weller, 1991). In androdioecious populations of $M$. annua (see below), sex appears to be determined to a large extent genetically; seed sampled from populations lacking males produced all cosex progeny, whereas seed from androdioecious populations produced both male and cosex progeny when grown under uniform conditions (Pannell, 1995). However, male frequency correlated significantly with stand density in two populations in the field, suggesting that there may also be an environmental component governing sex expression in the species.

In this paper, I attempt to resolve the environmental and genetic components of sex determination in M. annua. In particular, I ask whether gender in androdioecious $M$. annua is inherited by a simple 'active-Y' mechanism (Meagher, 1988), such that maleness is determined by the expression of a single or several linked dominant alleles (as has been demonstrated for polyploid species of Rumex (Love \& Kapoor, 1967)), or whether a more complex system of inheritance is involved. I also aim to test the hypothesis experimentally that stand density affects male frequency.

\section{The species}

Mercurialis annua L. s.l. (Euphorbiaceae) is a ruderal colonizer of pan-European distribution. Diploid $(2 n=16)$ populations are exclusively dioecious and are widespread across central and western Europe. Polyploids occur around the western Mediterranean in southern Europe and north Africa and are largely monoecious (Durand, 1963; Durand \& Durand, 1992). In the genus Mercurialis, all seven species are dioecious (apart from $M$. annua polyploids), and cosexuality would thus appear to be a derived trait (Tutin et al., 1968; Valdes et al., 1987). In southern Spain, southern Portugal and northern Morocco, hexaploid androdioecious populations of $M$. annua are widespread, with males co-occurring with monoecious plants (cosexes) at frequencies of less than about 30 per cent (Pannell, 1995, 1997a,b).

The physiology and genetic system of sex determination in diploid dioecious $M$. annua (i.e. where only males and females occur) have been characterized by Durand and coworkers (Louis et al., 1990; Durand \& Durand, 1991b). They have concluded that dioecy is controlled by three diallelic nuclear loci, A, B1 and B2, segregating independently in the nucleus. For an individual to be phenotypically male, it must possess a dominant $A$ allele with at least one dominant $B$ allele (i.e. either $B 1$ or $B 2$ ). An individual with a dominant $A$ allele but homozygous recessive at both $\mathrm{B}$-loci is phenotypically female, as are all genotypes homozygous recessive at the A-locus. Thus, for the $\mathrm{A}, \mathrm{B} 1$ and $\mathrm{B} 2$ loci, respectively, genotypes $(A /-, B 1 /-,-/-)$ and $(A /-,-/-, B 2 /-)$ are phenotypically male, whereas genotypes $(A /-, b 1 / b 1$, $b 2 / b 2)$ and $(a / a,-/,-/-)$ are phenotypically female (Durand \& Durand, 1991b).

In hexaploid androdioecious populations of $M$. annua, males have the same inflorescence morphology as those of the diploid populations (they have sessile staminate flowers arranged in tight spiral clusters along erect axillary peduncles). Hexaploid cosexes are similar in morphology to diploid females, except that their subsessile axillary pistillate flowers are surrounded basally by a tight spiral cluster of staminate flowers. Occasionally, cosexes are found with male pedunculate inflorescences in their lower leaf axils; the significance of these 'late cosexes' is interpreted in the discussion below. In both the diploids and the polyploids, plants produce flowers and fruits indeterminately in their leaf axils usually from the age of two weeks after germination until they die at the end of the growing season (Pannell, 1995, 1997b). Although it is likely that floral development and differentiation are governed by similar physiological gradients in both diploids and polyploids (Durand \& Durand, 1991b), nothing is yet known about the genetics of sex determination in polyploid populations of $M$. annua - neither whether the same (duplicated) sex-determining loci are responsible, nor how these may be expressed.

\section{Materials and methods}

Seed was collected for both the density and the breeding experiment in the spring of 1994 from a large androdioecious population of $M$. annua in the precincts of the Pabellón de Cuba in Sevilla, southern Spain. The frequency of males in the quadrats from which seed was taken ranged between about 10 per cent and 20 per cent (Pannell, 1995, 1997a). Plants were harvested and allowed to dry at room temperature to disperse their seeds. The seed was thoroughly mixed before subsampling for the experiments.

\section{Density experiment}

In late summer 1995, 12 seeds were sown in potting compost with a $1 \mathrm{~cm}$ covering of soil in each of 375 
pots $8 \mathrm{~cm}$ in diameter. Two weeks later, when seedlings had emerged and were at the cotyledon stage, their numbers were thinned to leave densities as follows: 200 pots with one plant; 100 pots with 2 plants; 50 pots with 4 plants; and 25 pots with 8 plants. (Increased numbers of pots were allocated to the lower density treatments so that sex ratios could be estimated between densities with equal precision.) The pots were arranged randomly on the glasshouse bench under natural light, and the appropriate densities were maintained for the duration of the experiment by the removal of any subsequent emergents. Plants were harvested after 8 weeks, approximately a month after they started flowering, and the numbers of males, pure cosexes and late cosexes were scored for each density.

\section{Breeding experiment}

In the summer of 1994, 500 plants were grown in potting compost in individual pots $(12 \mathrm{~cm}$ diameter) in the glasshouse under natural light until their sex could be determined 3 weeks after sowing. Eight plants were then moved into each of 13 individual pollen-proof growth boxes, in which they continued to grow and could mate amongst themselves. In six of the growth boxes, all eight plants were cosexes; in the other seven, four plants were cosexes and the other four were male. The plants were left to grow and reproduce for 10 weeks, at which point they were harvested, dried at room temperature, and their seeds were collected and stored. In the summer of 1995, the seeds produced by plants in each growth box were sown out into trays in potting compost, arranged randomly on the glasshouse bench, and were left to germinate and grow. At 8 weeks after sowing, plants were harvested and the numbers of males, pure cosexes and late cosexes were scored for each parental growth box.

\section{Results}

\section{Density experiment}

Table 1 presents the results of the density experiment in which individuals were scored as either males, late cosexes or cosexes. A chi-squared analysis of the proportion of pure cosexes (i.e. cosexes vs. males plus late cosexes) found no significant differences between the density treatments $\left(\chi_{3}^{2}=4.74\right.$, $P=0.19)$; cosexes occurred at a mean $( \pm$ SEM $)$ frequency of $73.15 \pm 1.60$ per cent across densities. As the frequency of cosexes was independent of density, cosexes were omitted in a subsequent analysis which included only the numbers of males and late cosexes. In this chi-squared test, the proportions of males and late cosexes differed highly significantly between densities $\left(\chi_{3}^{2}=38.65, P<0.001\right)$. There was a monotonic increase in the frequency of males with density and a general corresponding decrease in the proportion of late cosexes (Table 1). A linear regres-

Table 1 The numbers and percentages of males, late cosexes and cosexes of Mercurialis annua harvested 8 weeks after sowing in the density experiment. The composite for males and late cosexes, as well as the totals per density, are also shown

\begin{tabular}{lccccc}
\hline Density & Males & Late cosexes & $\begin{array}{c}\text { Males }+ \\
\text { late cosexes }\end{array}$ & Cosexes & Totals* \\
\hline 1 & 18 & 29 & 47 & 127 & 174 \\
& $(10.3 \%)$ & $(16.7 \%)$ & $(27.0)$ & $(73.0 \%)$ & \\
2 & 30 & 38 & 68 & 140 & 208 \\
& $(14.4 \%)$ & $(18.3 \%)$ & $(32.7)$ & $(67.3 \%)$ & \\
4 & 35 & 12 & 47 & 154 & 201 \\
& $(17.4 \%)$ & $(6.0 \%)$ & $(23.4)$ & $(76.6 \%)$ & \\
8 & 44 & 5 & 49 & 139 & 188 \\
& $(23.4 \%)$ & $(2.7 \%)$ & $(26.1)$ & $(73.9 \%)$ & \\
\hline
\end{tabular}

The density treatments were $1,2,4$ and 8 plants grown in $8 \mathrm{~cm}$ diameter pots. *Deviations from the planned number of plants per density $(=200)$ were largely caused by changes in the number of plants per pot through late emergents (as plants increased in size, it became difficult to see and thin out late emergents caused by the close packing of plants on the glasshouse bench); such pots were either assigned to the appropriate (higher) density, or excluded from the analysis. 
Table 2 The numbers of males, 'late cosexes', pure cosexes and immature (nonflowering Mercurialis annua) in the progeny of cosexes grown with or without males in growth boxes

\begin{tabular}{|c|c|c|c|c|c|}
\hline \multicolumn{2}{|c|}{ Number of males in treatment } & \multicolumn{4}{|c|}{ Number of individuals in progeny } \\
\hline Intended & Effective & Males & Late cosexes & Cosexes & Immature \\
\hline 0 & 0 & 0 & 0 & 165 & 0 \\
\hline 0 & 0 & 0 & 0 & 208 & 4 \\
\hline 0 & 0 & 0 & 0 & 371 & 43 \\
\hline 0 & 0 & 2 & 0 & 490 & 57 \\
\hline 0 & 0 & 2 & 0 & 195 & 7 \\
\hline 0 & 1 & 33 & 39 & 382 & 61 \\
\hline 0 & 1 & 4 & 3 & 475 & 52 \\
\hline 4 & 1 & 17 & 43 & 285 & 27 \\
\hline 4 & 2 & 17 & 54 & 331 & 30 \\
\hline 4 & 2 & 48 & 51 & 236 & 25 \\
\hline 4 & 3 & 76 & 17 & 241 & 16 \\
\hline 4 & 4 & 52 & 28 & 193 & 22 \\
\hline 4 & 4 & 18 & 15 & 112 & 12 \\
\hline
\end{tabular}

Either eight cosexes, or four males and four cosexes, were grown in each growth box together: the planned (intended) number of male parents is shown alongside the number of male parents actually counted at the time of harvest; the number of cosexes made up the full complement of eight plants per box.

sion of the proportions of males and late cosexes on $\log$ density was tested using the chi-squared test for trend (Armitage, 1971) and was highly significant $\left(\chi_{1}^{2}=34.86, P<0.001\right)$, with no significant departure from linearity $\left(\chi_{2}^{2}=1.46, P>0.25\right)$.

\section{Breeding experiment}

Over the course of the experiment, the number of males in some of the growth boxes changed from that intended; two boxes intended only for cosexes contained one male at the time of harvest, and five of the growth boxes ultimately contained fewer than the four males intended. The two cosex-to-male 'sex changes' were most likely caused by an error in sexing the plant initially (plants were small and had only just commenced budding). In contrast, although similar (but reversed) errors may have been partly responsible for the depletion of males from their intended number (four) in the mixed growth boxes, an actual gender change is likely to have played a role (see discussion below). This interpretation is foreshadowed by the fact that three of the eight cosex parents originally diagnosed as males turned out to be late cosexes.

Table 2 shows the numbers of males, late cosexes, pure cosexes and nonflowering (immature) individuals in the progeny of cosexes grown together in pollen-proof growth boxes either with or without males. Most striking is the almost complete lack of males and the total lack of late cosexes in the progeny of cosexes grown in the absence of males. By contrast, the progeny of cosexes grown in the presence of males always included both males and late cosexes (Table 2).

\section{Discussion}

\section{Males vs. pure cosexes}

In the breeding experiment, cosexes produced no male offspring unless males were present in the pollen-proof growth boxes. Although two of the growth boxes lacking males produced two male individuals in their progeny, these exceptions corresponded here to less than 1 per cent and 0.2 per cent of the progeny, respectively, and are consistent with the possibility of pollen contamination from outside the growth box during the course of the experiment (the boxes were opened every couple of days for watering).

These results are consistent with a single-locus inheritance of sex in $M$. annua hexaploids, with males having one or more dominant alleles for maleness, and pure cosexes being homozygous recessive, at the sex-determining locus. This interpretation holds for both disomic and hexasomic inheritance and suggests that sex is determined by a 
genetic system analogous to the active- $Y$ mechanism (Westergaard, 1958; Meagher, 1988). It is inconsistent with a system based on allele dosage (or a recessive allele for maleness), for which cosexes grown in the absence of males are likely to have segregated some male offspring.

If we assume that the same (A, B1 and B2) loci are responsible for genetic sex determination in $M$. annua hexaploids as in the diploids (Durand \& Durand, 1991b), and that cosexes represent a modified female phenotype, then the results presented here would be consistent with four different scenarios (Table 3): (1) dominant alleles at fixation for both $\mathrm{B}-\mathrm{loci}$, with the A-locus dimorphic (such that the presence of dominant $A$ determines maleness); (2) and (3) a dominant allele at fixation at the A-locus, and one of the B-loci at fixation for the recessive allele $b$, with the remaining B-locus dimorphic (such that the presence of a dominant $B$ determines maleness); and (4) the A-locus at fixation for the dominant allele $A$, and both of the B-loci potentially dimorphic (Table 3 ).

As yet, there is no information about the mode of sex inheritance in the other known androdioecious plant species, Datisca glomerata (Liston et al., 1990; Fritsch \& Rieseberg, 1992), Phillyrea angustifolia (Lepart \& Dommée, 1992; Traveset, 1994) and P. latifolia (Aronne \& Wilcock, 1994). However, the genetic system for sex determination in the notostracan Eulimnadia texana is well characterized (Sassaman, 1989, 1991; Otto et al., 1993; Sassaman \& Weeks, 1993) and contrasts with that in M. annua described here. Whereas maleness in $M$. annua is controlled by a dominant allele, in $E$. texana the allele for maleness is recessive, such that cosexes are either homozygous recessive or heterozygous at the sex-determining locus (Sassaman \& Weeks, 1993). Moreover, whereas cosexes in $M$. annua are both self-fertile and capable of outcrossing as male parents, $E$. texana cosexes are self-fertile only, with their male function being incapable of outcrossing (Otto et al., 1993).

\section{'Late cosexuality'}

The association between the lack of male progeny produced by cosexes in the absence of males and the lack of the late cosexes in the same progeny is a particularly interesting finding. One possible explanation is that there exists a gene for maleness which is only partially or subdominantly expressed as 'late cosexuality'. This would imply that such cosexes should segregate late cosexes in their own progeny, even when fertilized only by cosexual pollen, a prediction which still needs to be tested. I suggest, however, that late cosexuality in the population investigated here is more likely to result from a certain degree of lability in the sex determination system in $M$. annua, such that (at least some) genetic males have the ability to function cosexually, possibly in response to an environmental cue. This lability in sex expression would appear to be unidirectional, with only genetic males capable of altering their sex.

There are three reasons for this hypothesis. First, in the breeding experiment, genetic cosexes (i.e. the progeny of male-less crosses) were always pure cosexes, never bearing male peduncles in their lower leaf axils, whereas late cosexes only appeared in the progeny alongside pure males. If genetic cosexes possessed the ability to produce male peduncles as young plants, the complete absence of such individuals in the progeny of male-less parents would be inexplicable. Secondly, in the density experiment, male frequencies decreased at lower stand densities, and the frequency of late cosexes increased, whereas there was no difference in the frequency of pure cosexes between densities. This strongly implicates a density-dependent sex change between males and late cosexes, with genetic males becoming cosexual at low densities later in their development. Thirdly, physiological experiments on diploid dioecious $M$. annua plants have found that whereas males can be feminized by the exogenous application of certain plant growth substances (see below), females cannot

Table 3 Possible scenarios for the genetic system of sex determination in androdioecious hexaploid Mercurialis annua, assuming the same sex-determining loci as proposed by Durand (1991b); see text for explanation

\begin{tabular}{llllll}
\hline Scenario & A-locus & B1-locus & B2-locus & \multicolumn{1}{c}{ Phenotype } \\
\hline 1 & Dimorphic & $B 1$ fixed & $B 2$ fixed & Any $A \rightarrow$ male & Only $a \rightarrow \operatorname{cosex}$ \\
2 & $A$ fixed & Dimorphic & $b 2$ fixed & Any $B 1 \rightarrow$ male & Only $b 1 \rightarrow \operatorname{cosex}$ \\
3 & $A$ fixed & $b 1$ fixed & Dimorphic & Any $B 2 \rightarrow$ male & Only $b 2 \rightarrow \operatorname{cosex}$ \\
4 & $A$ fixed & Dimorphic & Dimorphic & Any $B \rightarrow$ male & Only $b \rightarrow \operatorname{cosex}$ \\
\hline
\end{tabular}


be masculinized (Durand \& Durand, 1991b), suggesting the potential for an asymmetry in sex choice in the species.

It is noteworthy that because males can become cosexual and self-fertilize (or cross with other males), their male progeny could carry more than one dominant allele for maleness (in contrast to the dioecious situation where males would be heterogametic). This suggests the possibility that maledetermining allele dosage could determine a male's potential for sex choice. It is certainly interesting that in diploid $M$. annua, differences in sex allele dosage in males are expressed in terms of differing sensitivities to exogenous feminizing hormones (Durand \& Durand, 1991b), and it is conceivable not only that the same might apply to androdioecious hexaploids but also that endogenous levels of such sex-affecting hormones could be altered in response to the environment.

\section{Environmental sex determination}

This study leaves little doubt that there is an environmental component in the determination of sex in androdioecious $M$. annua. Once seedlings emerged in the density experiment, there was no mortality, thus discounting the possibility of differential mortality. Nor is it conceivable that differential germination of the sexes occurred, as seeds were sown at the same densities initially.

The switch to cosexuality by more males at lower than at higher densities appears to conform to the generalization for plants that favourable growing conditions (here, low densities) enhance female sex expression (reviewed by Meagher, 1988). This might be expected, given the greater burden of costs associated with the female function during seed and fruit maturation (Kohn, 1989). Such an hypothesis, however, predicts a similar shift towards male function in the sex allocation of cosexes (as found in monoecious spinach plants; Freeman \& Vitale, 1985), whereas in $M$. annua the reverse occurs (at high experimental densities, cosexual sex allocation shifts towards the female function; Pannell, 1995, 1997a). This suggests that sex choice in genetic males and sex allocation in cosexes are affected differently by factors other than merely cost constraints. It is likely that these patterns are adaptive responses to selection for reproductive assurance during colonization (see below).

\section{Implications for the maintenance of androdioecy}

Androdioecy is an extremely rare mating system
(Charlesworth, 1984; Liston et al., 1990; Fritsch \& Rieseberg, 1992), and theoretical studies have shown that it is unlikely to be maintained in nature. One reason for this is that males can only coexist with cosexes if they are more than twice as fertile as the cosex male function, a fertility threshold which increases in partially selfing populations (Lloyd, 1975; Charlesworth \& Charlesworth, 1978). Moreover, in a colonizing species such as Mercurialis annua, self-fertile cosexes will enjoy the important advantage over males of reproductive assurance during colonization, because they can start a new colony by self-fertilization when isolated from prospective mates (Baker, 1955, 1967; Jarne \& Charlesworth, 1993; Pannell, 1997c). The persistence of males with cosexes in populations of $M$. annua would thus seem even more unlikely.

There appear to be at least two reasons for the maintenance of androdioecy in $M$. annua. First, males produce between four and 10 times as much pollen as cosexes, well above the fertility threshold required for their maintenance, even with high selfing rates (Pannell, 1995, 1997b). And secondly, the ability of (at least some) males to function as cosexes at low densities, as demonstrated in this study, is likely to mitigate against selection for reproductive assurance which would otherwise favour pure cosexuality. Clearly, such sexually labile males will enjoy the advantage of high outcrossing success in the company of cosexual neighbours, while at the same time not risking total reproductive failure as sole colonizers.

\section{Acknowledgements}

I am grateful to Alan Grafen, Andrew Lack, Deborah Charlesworth and two anonymous reviewers for helpful comments on the manuscript, and to Phil Smith and Elliot Rhymes for tending to the plants. My thanks go also to the staff and students of the Department of Botany, the University of Seville, for logistical support in the field. This work was carried out while I was supported in Oxford by a scholarship from the Association of Commonwealth Universities.

\section{References}

ARMITAGE, P. 1971. Statistical Methods in Medical Research, 1st edn. Blackwell Scientific Publications, Oxford. ARONNE, G. AND WILCOCK, C. C. 1994. Reproductive characteristics and breeding system of shrubs in the Mediterranean region. Funct. Ecol., 8, 69-76.

BAKER, H. G. 1955 . Self-compatibility and establishment 
after 'long-distance' dispersal. Evolution, 9, 347-348.

BAKER, H. G. 1967. Support for Baker's Law - as a rule. Evolution, 21, 853-856.

CHARLESWORTH, D. 1984. Androdioecy and the evolution of dioecy. Biol. J. Linn. Soc., 22, 333-348.

CHARLESWORTH, D. AND CHARLESWORTH, B. 1978. A model for the evolution of dioecy and gynodioecy. Am. Nat., 112, 975-997.

Charnov, E. L. 1982. The Theory of Sex Allocation. Princeton University Press, Princeton, NJ.

DURAND, B. 1963. Le complexe Mercurialis annua L. s.l.: une étude biosystematique. Ann. Sci. Nat. Bot., 12, 579-736.

DURAND, B. AND DURAND, R. 1991a. Male sterility and restored fertility in annual mercuries: relations with sex differentiation. Plant Sci., 80, 107-118.

DURAND, B. AND DURAND, R. 1991b. Sex determination and reproductive organ differentiation in Mercurialis. Plant Sci., 80, 49-66.

DURAND, R. AND DURAND, в. 1992. Dioecy, monoecy, polyploidy and speciation in annual Mercuries. Bull. Soc. Bot. Fr. Lett. Bot., 139, 377-399.

FREEMAN, D. C. AND VITALE, J. J. 1985. The influence of environment on the sex ratio and fitness of spinach. Bot. Gaz., 146, 137-142.

FRITSCH, P. AND RIESEBERG, L. H. 1992. High outcrossing rates maintain male and hermaphrodite individuals in populations of the flowering plant Datisca glomerata. Nature, 359, 633-636.

GOUYON, P.-H. AND COUVET, D. 1987. A conflict between two sexes, females and hermaphrodites. In: Stearns, S. C. (ed.) The Evolution of Sex and its Consequences, pp. 245-261. Birkhäuser Verlag, Basel.

IRISH, E. E. AND NELSON, T. 1989. Sex determination in monoecious and dioecious plants. Pl. Cell, 1, 737-744.

JARNE, P. AND CHARLESWORTH, D. 1993. The evolution of the selfing rate in functionally hermaphrodite plants and animals. Ann. Rev. Ecol. Syst., 24, 441-466.

KOHN, J. R. 1989. Sex ratio, seed production, biomass allocation, and the cost of female function in Curcurbita foetidissima HBK (Curcurbitaceae). Evolution, 43, 1424-1434.

LEPART, J. AND DOMMÉE, в. 1992. Is Phillyrea angustifolia L. (Oleaceae) an androdioecious species? Bot. J. Linn. Soc., 108, 375-387.

Liston, A., RIESEBerG, L. H. AND elias, T. s. 1990. Functional androdioecy in the flowering plant Datisca glomerata. Nature, 343, 641-642.

LLOYD, D. G. 1975. The maintenance of gynodioecy and androdioecy in angiosperms. Genetica, 45, 325-339.

LLOYD, D. G. AND BAWA, K. S. 1984. Modification of the gender of seed plants in varying conditions. Evol. Biol., 17, 255-336.

LOUis, J. P., AUGUR, C. AND Teller, G. 1990. Cytokinins and differentiation processes in Mercurialis annua. Genetic regulation, regulations with auxins, indoleacetic acid oxidases, and sexual expression patterns. $P l$. Physiol., 94, 1535-1541.

LOVE, A. AND KAPOOR, в. M. 1967. A chromosome atlas of the collective genus Rumex. Cytologia, 32, 328-342.

MEAGHER, T. R. 1988. Sex determination in plants. In: Lovett Doust, J. and Lovett Doust, L. (eds) Plant Reproductive Ecology: Patterns and Strategies, pp. 125-138. Oxford University Press, New York.

otTo, s. P., SASSAMAN, C. AND FELdMAN, M. W. 1993. Evolution of sex determination in the conchostracan shrimp Eulimnadia texana. Am. Nat., 141, 327-337.

PANNELL, J. R. 1995. Models of Androdioecy and Studies on Mercurialis annua $L$. D.Phil. Thesis, University of Oxford.

PANNELL, J. R. 1997a. Variation in sex ratios and sex allocation in androdioecious Mercurialis annua. J. Ecol. (in press).

PANNELL, J. R. 1997b. Widespread functional androdioecy in Mercurialis annua L. (Euphorbiaceae). Biol. J. Linn. Soc. (in press).

PANNELL, J. R. 1997c. The maintenance of gynodioecy and androdioecy in a metapopulation. Evolution (in press).

REBOUD, X. AND ZEYL, C. 1994. Organelle inheritance in plants. Heredity, 72, 132-140.

SAKAI, A. K. AND WELLER, S. G. 1991. Ecological aspects of sex expression in subdioecious Schiedea globosa (Caryophyllaceae). Am. J. Bot, 78, 1280-1288.

SASSAMAN, C. 1989. Inbreeding and sex ratio variation in female-biased populations of a clam shrimp, Eulimnadia texana. Bull. Mar. Sci., 45, 425-432.

SASSAMAN, C. 1991. Sex ratio variation in female-biased populations of Notostracans. Hydrobiologia, 212, 169-179.

SASSAMAN, C. AND WEeks, s. c. 1993. The genetic mechanism of sex determination in the conchostracan shrimp Eulimnadia texana. Am. Nat., 141, 314-328.

TRAVESET, A. 1994. Reproductive biology of Phillyrea angustifolia L. (Oleaceae) and effect of galling-insects on its reproductive output. Bot. J. Linn. Soc., 114, 153-166.

TUTIN, T. G., HEYWOOD, V. H., BURGES, N. A., MOORE, D. M., VAlentine, D. H., WAlTERS, S. M. AND WebB, D. A. 1968. Flora Europaea, vol. 2. Cambridge University Press, Cambridge.

VALDES, B., TALAVERA, S. AND FERNANDEZ-Galiano, E. 1987. Flora Vascular de Andalucía Occidental, vol. 2. Ketres Editora, Barcelona.

VAN DAMME, J. M. M. 1991. A restorer gene in gynodioecious Plantago coronopus subject to selection in the gametophytic and seedling stage. Heredity, 66, 19-27.

WESTERGAARD, M. 1958. The mechanism of sex determination in flowering plants. Adv. Genet., 9, 217-281. 